\section{Uncoupling NMDA receptor interactions}

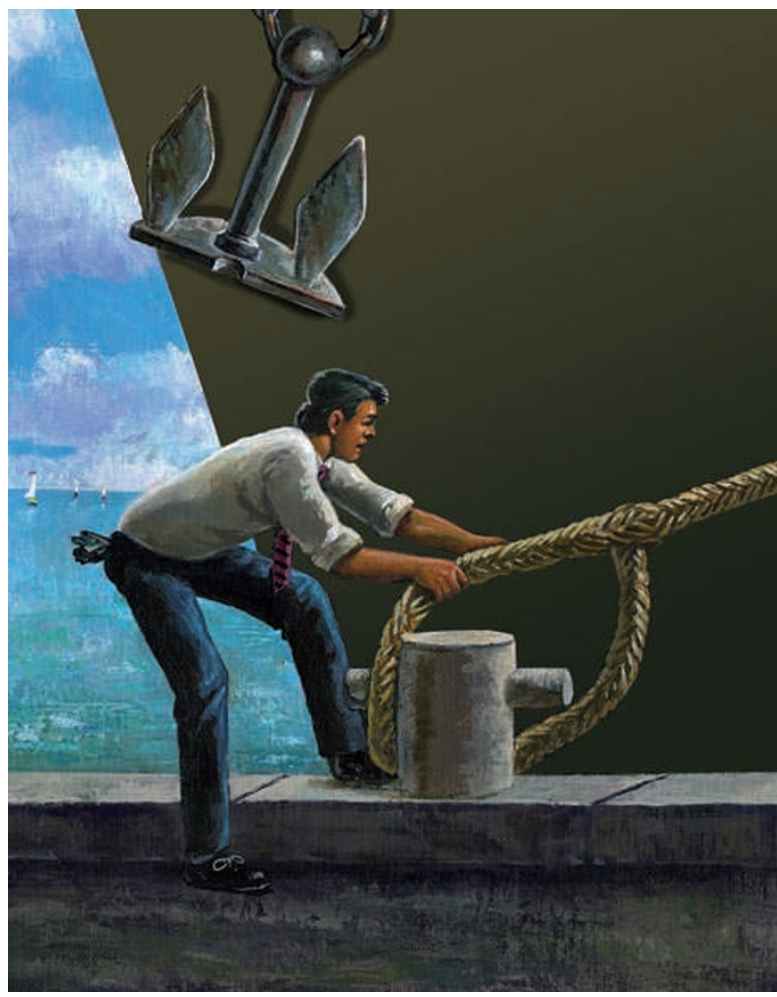

Chronic pain hypersensitivity depends on the upregulation of NMDAR ( $N$-methyl-D-aspartate receptor) activity, but blocking this receptor is linked to respiratory and locomotory side effects. Now, writing in Nature Medicine, Liu and colleagues describe a strategy that suppresses pain - without blocking NMDARs - which involves uncoupling NMDARs from the protein tyrosine kinase Src, a key enhancer of NMDAR function.

The authors had previously synthesized peptides covering a region in the Src-unique domain that is crucial for its interaction with NADH dehydrogenase subunit 2 (ND2), the adapter protein that anchors Src within the NMDAR complex. In the current study, a peptide consisting of amino acids $40-49$ of Src (Src40-49) disrupted the interaction between Src and the NMDAR complex by inhibiting the binding of the kinase to ND2, without affecting Src catalytic activity. In cultured neurons in which NMDARs are tonically upregulated by Src, the peptide reduced NMDAR-mediated currents.

Next, the authors investigated the in vivo effects of a membranepermeable peptide, Src40-49Tat. In a rat inflammatory pain model, pretreatment with Src40-49Tat suppressed NMDAR-mediated pain behaviours, without causing observable changes in peripheral inflammation, basal sensory thresholds, locomotor performance, learning and memory, or cardiorespiratory functions.

In a model of established inflammatory pain, Src40-49Tat inhibited thermal and mechanical hypersensitivity, with both effects lasting around 2 hours. When Src40-49Tat was tested on neuropathic pain behaviours, the peptide reversed nerve-injury-induced mechanical hypersensitivity, and when administered intrathecally, the antinociceptive effect persisted for the 5-hour testing period. In this model of peripheral nerve injury, Src40-49Tat also reduced acetone-induced coldsensitization behaviours.

Suppression of inflammatory and neuropathic pain behaviours by Src40-49Tat was not observed in $\mathrm{Src}^{-/-}$mice, indicating that the effect was Src-dependent. Furthermore, it was shown that the suppression of NMDA-mediated pain behaviour occurred within the spinal cord; in this area, Src40-49Tat disrupted the Src-ND2 interaction and reduced the increase in tyrosine phosphorylation of the NR2B subunit of NMDARs that occurred in response to the pain-inducing agents.

So, disrupting the Src-ND2 interaction produces NMDARmediated analgesia while avoiding the undesirable effects of blocking NMDAR function, and does not inhibit the catalytic activity of Src. Although this work is at an early stage, it could represent a new strategy for the treatment of chronic pain and potentially other NMDARdependent CNS pathologies.

Charlotte Harrison Senior Editor Nature Reviews Drug Discovery

ORIGINAL RESEARCH PAPER Liu, X. J. et al. Treatment of inflammatory and neuropathic pain by uncoupling Src from the NMDA receptor complex. Nature Med. 14, 1325-1332 (2008) 